University of Windsor

Scholarship at UWindsor

\title{
Theoretical proposal for a biosensing approach based on a linear array of immobilized gold nanoparticles
}

\author{
S.M.H. Rafsanjani \\ T. Cheng \\ S. Mittler \\ Chitra Rangan \\ University of Windsor
}

Follow this and additional works at: https://scholar.uwindsor.ca/physicspub

Part of the Engineering Physics Commons

\section{Recommended Citation}

Rafsanjani, S.M.H.; Cheng, T.; Mittler, S.; and Rangan, Chitra. (2010). Theoretical proposal for a biosensing approach based on a linear array of immobilized gold nanoparticles. Journal of Applied Physics, 107 (9), 094303-1-094303-6.

https://scholar.uwindsor.ca/physicspub/1

This Article is brought to you for free and open access by the Department of Physics at Scholarship at UWindsor. It has been accepted for inclusion in Physics Publications by an authorized administrator of Scholarship at UWindsor. For more information, please contact scholarship@uwindsor.ca. 


\section{AIP Appinied Physics}

\section{Theoretical proposal for a biosensing approach based on a linear array of immobilized gold nanoparticles}

S. M. Hashemi Rafsanjani, T. Cheng, S. Mittler, and C. Rangan

Citation: J. Appl. Phys. 107, 094303 (2010); doi: 10.1063/1.3369440

View online: http://dx.doi.org/10.1063/1.3369440

View Table of Contents: http://jap.aip.org/resource/1/JAPIAU/v107/i9

Published by the American Institute of Physics.

\section{Related Articles}

New Products

Rev. Sci. Instrum. 83, 099501 (2012)

Non-destructive imaging of an individual protein

Appl. Phys. Lett. 101, 093701 (2012)

Ultrafast excited state dynamics of spirilloxanthin in solution and bound to core antenna complexes: Identification of the S and T1 states

JCP: BioChem. Phys. 6, 08B610 (2012)

Ultrafast excited state dynamics of spirilloxanthin in solution and bound to core antenna complexes: Identification of the $\mathrm{S}^{*}$ and $\mathrm{T} 1$ states

J. Chem. Phys. 137, 064505 (2012)

Magneto-optic surface plasmon resonance optimum layers: Simulations for biological relevant refractive index changes

J. Appl. Phys. 112, 034505 (2012)

\section{Additional information on J. Appl. Phys.}

Journal Homepage: http://jap.aip.org/

Journal Information: http://jap.aip.org/about/about_the_journal

Top downloads: http://jap.aip.org/features/most_downloaded

Information for Authors: http://jap.aip.org/authors

\section{ADVERTISEMENT}
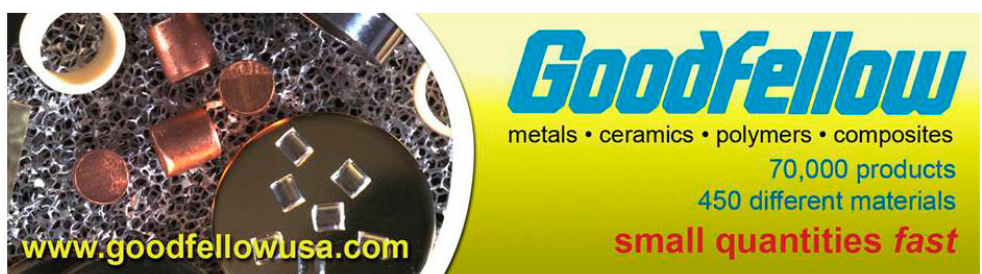


\title{
Theoretical proposal for a biosensing approach based on a linear array of immobilized gold nanoparticles
}

\author{
S. M. Hashemi Rafsanjani, ${ }^{1, a)}$ T. Cheng, ${ }^{1}$ S. Mittler, ${ }^{2}$ and C. Rangan ${ }^{1, b)}$ \\ ${ }^{1}$ Department of Physics, University of Windsor, Ontario, N9B 3P4, Canada \\ ${ }^{2}$ Department of Physics and Astronomy, University of Western Ontario, London, Ontario, N6A 3K7, Canada
}

(Received 6 April 2009; accepted 21 February 2010; published online 3 May 2010)

\begin{abstract}
We propose a sensing mechanism for detection of analytes that can specifically recognized. The sensor is based on closely-spaced chains of functionalized gold nanoparticles (NPs) immobilized on a waveguide surface, with the signal detected by evanescent waveguide absorption spectroscopy. The localized surface plasmon spectrum of a linear array of closely-spaced, hemispherical gold NPs is calculated using the discrete dipole approximation. The plasmon band is found to broaden to a nanowirelike spectrum when a dielectric coating is put on the particles, and the light polarization is along the NP chain. The origin of this broadening is shown to be the polarization-dependent overlap of the evanescent fields of adjacent NPs upon application of the dielectric coating. These features suggests a mechanism for biosensing with an improved sensitivity compared with traditional NP biosensor methods. (C) 2010 American Institute of Physics. [doi:10.1063/1.3369440]
\end{abstract}

\section{INTRODUCTION}

Gold NPs (GNPs) are good candidates for implementing biosensing methods ${ }^{1}$ because they combine narrow and sensitive localized surface plasmon resonance (LSPR) spectra $^{2,3}$ with the strong binding affinity of thiol groups to gold, ${ }^{4}$ allowing the immobilization of a recognition site for a particular analyte molecule. ${ }^{5}$ The effects of size, ${ }^{6}$ shape, ${ }^{7}$ substrate, ${ }^{8}$ and plasmon coupling within clusters ${ }^{9}$ on the gold LSPR spectrum have been well studied. An excellent review of refractive index sensing using noble-metal NPs can be found in Ref. 10. GNPs can also be immobilized on a surface for planar biosensing ${ }^{11}$ offering an increased sensitivity at smaller sample volumes and the potential of multiplexing and parallelization.

We present the theoretical principle behind a biosensor that uses a chain of hemispherical GNPs (HGNPs) immobilized on a waveguide surface, and spectral detection via evanescent wave absorption spectroscopy (EWAS). ${ }^{12,13}$ The presence of a small amount of dielectric material can be detected by a large change in the EWAS spectra. In the EWAS method, the NPs are located in the evanescent field of the waveguide which excites the localized surface plasmons in the NPs. The evanescent field can have two polarizationscorresponding to the transverse electric (TE) and transverse magnetic (TM) waveguide modes. Thus the NPs can be addressed with two polarizations in contrast to transmission absorption spectroscopy. ${ }^{14}$ By modeling a detected analyte layer by a thin dielectric coating, we show that a specific recognition reaction can be detected by the large change in the optical response of the HGNP chain - the broadening of the NP LSPR peak to a broad nanowirelike spectrum.

Recently, Aliganga et al. ${ }^{15}$ and Rooney et al. ${ }^{16}$ have shown that by immobilizing NPs on a waveguide surface and

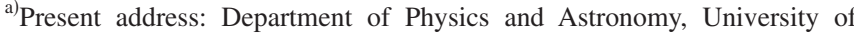
Rochester, NY, USA

${ }^{b)}$ Electronic mail: rangan@uwindsor.ca.
}

using EWAS (Refs. 12 and 13) for detection of spectral information, it is possible to experimentally distinguish between bare GNPs deposited on a surface, and the same NPs coated with an ultrathin, organic, dielectric layer. The immobilized NPs were coupled optically into clusters by the application of the dielectric coating. This clustering led to the appearance of a second peak in the absorption spectrum - the optical clustering process-only when the polarization direction was chosen to be in the plane of the NPs. The present theoretical work investigates the possibility of increasing the sensitivity of the above process by placing HGNPs in a regularly-spaced chain, and determines the EWAS spectrum of a chain of regularly-spaced dielectrically coated HGNPs. Note that this study is fundamentally different from the NP array biosensors ${ }^{17-19}$ that use interferometric effects to produce narrow plasmon bands and significant field enhancements.

We study a linear chain of closely-spaced HGNPs as a model of the experiment where a row of GNPs are immobilized on a waveguide's surface. In such a chain (with interparticle separation much smaller than the wavelength of light), we show that by coating the NPs with a dielectric layer that models the bound analyte, the EWAS spectrum in the $s$-polarization changes from the narrow single-particle LSPR spectrum to a broad nanowirelike spectrum. This effect is shown to be due to the polarization-dependent overlap of the evanescent electric fields of the individual NPs induced by the coating. Our study of spherical and hemispherical NP chains indicates that hemispherical NP chains are better suited as biosensors than spherical NP chains. We also present a measure of biosensor sensitivity that is an improvement over present measures that are keyed to the peak LSPR wavelength shift as function of analyte refractive index. The next step in the implementation of the proposed biosensor will be a specific recognition reaction of an analyte that can 
be detected by the large change in the optical response due to the build up of organic material on the GNP chains - these experiments are in progress.

\section{METHOD}

In this work, we study the influence of a dielectric coating on a linear chain of very closely-spaced (spacing $\ll \lambda$ ) gold nanohemispheres on the plasmon spectrum. Markel ${ }^{19}$ showed that if the spacing between particles in a linear chain is much smaller than the wavelength of the radiation, then the wavelength of the plasmon resonance is dominated by the near-zone (i.e., $1 / r^{3}$ ) interaction. Zhao et al. ${ }^{18}$ calculated the plasmon band for a planar lattice of spherical silver NPs and found that as the spacing between the particles was decreased to be much smaller than the wavelength of the incident light, the peak of the UV-vis plasmon spectrum redshifts, and the width of the plasmon band broadens. We show that a similar broadening of the spectrum can be produced by adding a dielectric coating on a chain of HGNPs. The physical origin of the broadening is shown to be the overlap of the electric fields between adjacent NPs in a chain that occurs when the particles are coated by a dielectric material (modeling the analyte layer).

To calculate the LSPR spectrum of a chain of HGNPs, we solve Maxwell's equations for the configuration when white light is incident on a chain of GNPs along the surface upon which the particles are immobilized, and perpendicular to the chain direction (see Fig. 1 top panel). The light polarization is then either along the surface (along the interparticle axis) $s$ or perpendicular to the surface (and to the interparticle axis) $p$. The extinction spectrum as well as the electric field around the NPs are calculated using the well-known discrete dipole approximation (DDA).$^{20,21}$ In this method, a continuum target is modeled by a lattice of $N$ discrete dipoles at points $r_{i}$, and a polarizability $\alpha_{i}$ is assigned to each dipole. The $\alpha_{i}$ 's are chosen so that for large $N$ the lattice has the same dispersion relation as a bulk continuum target. The dielectric constants for gold in the optical wavelength range is taken from Palik's handbook. ${ }^{22}$ The validity criterion of the method is that the target lattice should be dense enough so that the phase change from one dipole to its neighbor is less than $1 \mathrm{rad}$; i.e., the lattice spacing is much smaller than the wavelength. ${ }^{21}$ This criteria implies that $|m| k d \ll 1$, where $m$ is the refractive index of the target and $d$ is the lattice spacing. The computations were done using a FORTRAN implementation of DDA introduced by Draine and Flatau ${ }^{21}$ that has been used for nanoplasmonics calculations by several groups. ${ }^{7,16,23,24}$ We calculate the extinction, absorption, and scattering efficiencies, as well as the magnitude of electric field at the site of each dipole, details are given in Refs. 7 and 16.

In this study, the substrate (waveguide surface) is not explicitly modeled. That is, the substrate's refractive index is taken to be the same as that of the ambient medium. This is justified because in the planned experiments, the GNPs are not in direct contact with substrate, and are immobilized via silane chemistry, ${ }^{25}$ that is, the NPs are tethered to the waveguide surface. From investigations on substrate effects, ${ }^{10,26}$ it

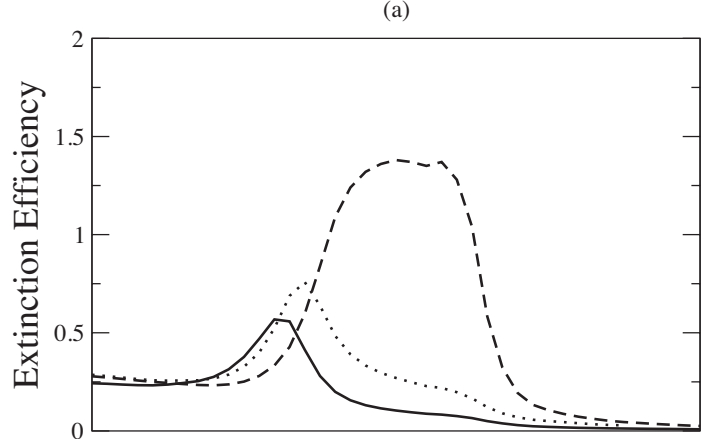

(b)

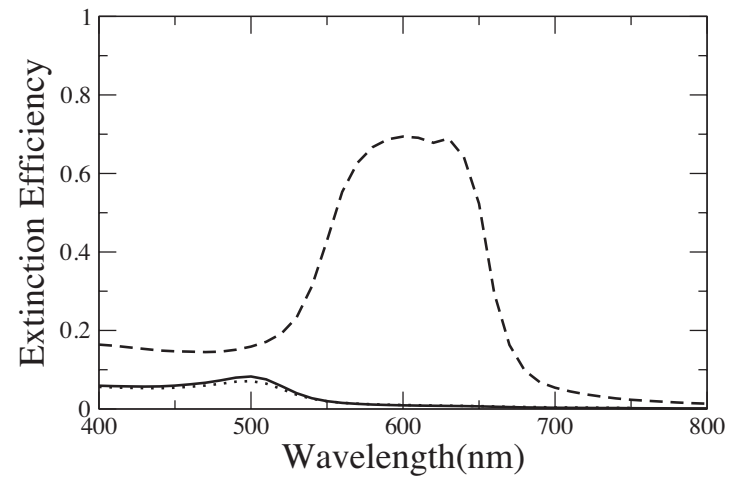

FIG. 1. Effect of contact between the GNPs and a substrate. Extinction spectra of a HGNP array excited by light propagating along the waveguide surface and perpendicular to the chain. Light polarization is either (a) along the interparticle axis ( $s$-polarization) or (b) perpendicular to the waveguide surface ( $p$-polarization). Spectra are shown when there is no substrate (solid lines), when the NPs are $7 \mathrm{~nm}$ away from the substrate (dotted lines), and when the NPs are in contact with the substrate (dashed lines). The extinction spectra show a dramatic change when the NPs are put on a silicon dioxide substrate, but only a small change when the NPs are $7 \mathrm{~nm}$ away from the substrate. The radius of each hemispherical NP is $7 \mathrm{~nm}$ and the interparticle spacing is $17.5 \mathrm{~nm}$ center to center.

is clear that substrate effects become significant when there is direct contact between the NP and the substrate, and when the incident electric field is polarized perpendicular to the substrate. In our proposed biosensor platform the GNPs are not in direct contact with substrate, and the electric field is parallel to the surface. Figure 1 shows a DDA calculation using the methodology of Ref. 27 that confirms this hypothesis, that is when the HGNP array is a distance of $7 \mathrm{~nm}$ away from the surface, the effect on the LSPR spectrum is a small $(10 \mathrm{~nm})$ shift in the peak of the spectrum. (In the experiment, the spacing between the GNPs and the substrate is of the order of $10 \mathrm{~nm}$.) Note that in this sensor scheme, we are not looking for peak wavelength shifts, but for the ratio of peak heights. Therefore for the calculations in the rest of the paper, we consider a chain of GNPs in a matrix with the refractive index of air.

In the EWAS configuration, the NPs are illuminated by the evanescent field of the waveguide. This field propagates parallel to the waveguide surface with its polarization along the waveguide mode polarization. Since the field decays over several microns away from the waveguide surface, and the NPs are only of the order of $10 \mathrm{~nm}$, we can treat the incident field as a plane wave in the calculations. We approximate the TE modes by applying $s$-polarized light (electric field pointing along the waveguide surface) and the TM modes by ap- 
(a)

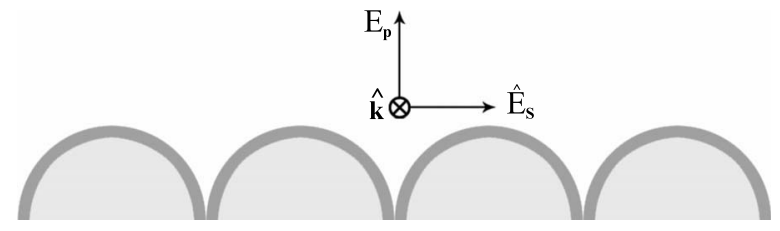

(a)

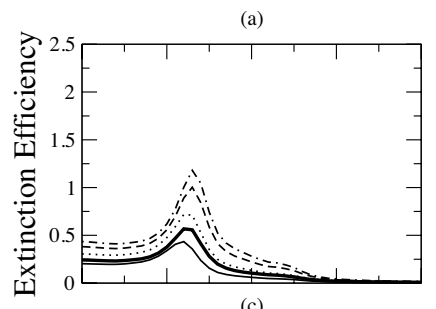

(c)

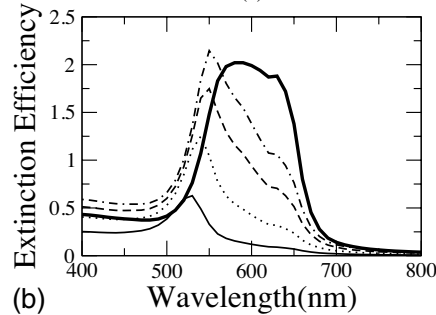

(b)
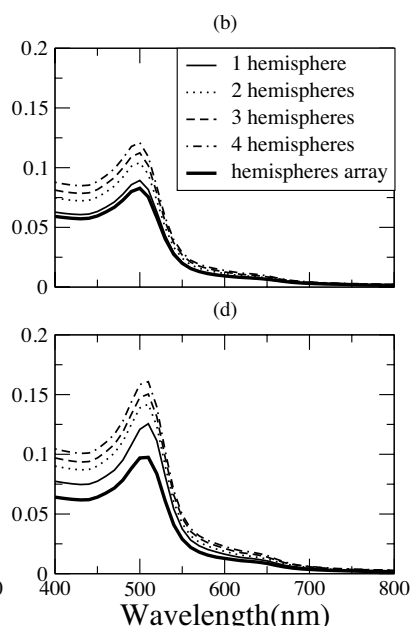

FIG. 2. Extinction spectra of HGNP chains with different numbers of particles excited by light propagating along the waveguide surface and perpendicular to the chain. Light polarization is either along the interparticle axis ( $s$-polarization) - left two subgraphs, or perpendicular to the waveguide surface ( $p$-polarization) - right two subgraphs. The extinction spectra change from (a) and (b) when the NPs are bare to (c) and (d) when the NPs are coated by a dielectric analyte layer. The radius of each hemispherical NP is $7 \mathrm{~nm}$ and the thickness of the coating layer is $1.75 \mathrm{~nm}$. The interparticle spacing is $17.5 \mathrm{~nm}$ center to center.

plying $p$-polarized light (electric field pointing perpendicular to the waveguide surface) to the NP chain - the chain is perpendicular to the field propagation. The small E-field component oscillating in the direction of the k-vector in TM modes is not taken into account since it does not affect the main results of this paper (since the interparticle axis is always perpendicular to the propagation vector). Note that the plasmons do not couple to the waveguide modes, and the NPs are merely in the evanescent field of the waveguide. Therefore it is unnecessary to model the waveguide as well.

\section{RESULTS AND DISCUSSION}

We now present numerical results of the calculation of the extinction spectra of a chain of 2,3,4, and an infinite number of bare and dielectrically coated HGNPs. We consider a chain of HGNPs of $14 \mathrm{~nm}$ diameter and center-tocenter separation of $17.5 \mathrm{~nm}$. The analyte coating is modeled by a concentric $1.75 \mathrm{~nm}$ layer of a dielectric material with refractive index $n=1.45$ that covers only the exposed side of the NPs as shown in Fig. 2. This is because, in the experiment, the recognition sites for the analyte are situated only on the hemispherical surface of the nanoparticle (NP), whereas the planar surface of the NP is tethered to the waveguide. In Fig. 2, the upper two panels show the spectra of the bare NP chain, and the lower two panels show the spectra for the analyte-coated chain. The spectra for $s$-polarized light are on the left, and those for $p$-polarized light are on the right.

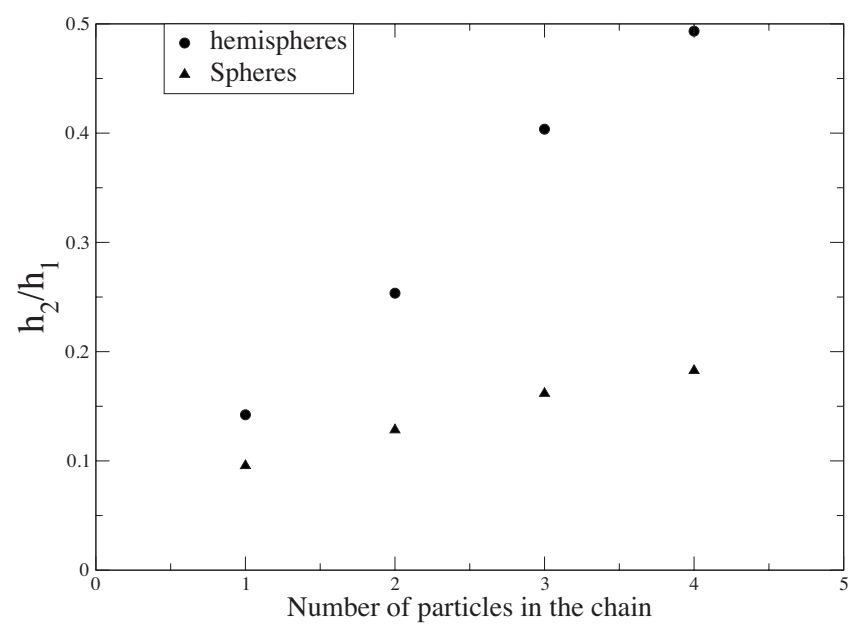

FIG. 3. The relative height of the second peak at $\sim 630 \mathrm{~nm}$ to the first peak $\sim(530-550 \mathrm{~nm})$ vs the number of particles in the chain

When coated by an analyte, the LSPR spectrum of the NP chain shows two changes. The first is that the peak of the spectrum shifts by $10-20 \mathrm{~nm}$ to the red, and this can be attributed to the increase in size of the NPs and an increase in the ambient refractive index. The second change is the appearance of a second peak at around $630 \mathrm{~nm}$ that appears only when the light polarization is along the inter particle axis (s-polarization). The shift in the first peak increases with increasing number of NPs in the chain but the location of the second peak is not as sensitive to the number of particles in the chain as the first peak. Interestingly, the ratio of peak heights $\left(h_{2} / h_{1}\right)$ increases as the number of the particles in the chain increases as seen in Fig. 3. For a single hemisphere the ratio is 0.14 and it increases to 0.5 for a chain of four hemispheres. Thus, the presence of a dielectric coating is detected by the change in the spectrum from a narrow NP-like spectrum to a broad nanowirelike spectrum. The sensitivity of detection is determined by the ratio of the height of the optical clustering peak to the height of the dipole peak.

\section{A. Effect of NP shape on LSPR spectra}

It is worth noting that this sensing mechanism is not as effective when a chain of spherical NPs are used. Figure 4 shows that in comparison to the hemispherical NP chain, a chain of spherical NPs simply does not produce the pronounced spectral feature at $630 \mathrm{~nm}$. In contrast to the hemispherical chain, the relative height of the cross-talk peak and the single particle peak does not change very much for spheres (see Figs. 3 and 4). This can be explained by noting that in the case of a hemisphere we have a broken symmetry and sharp edges where the particles are attached to the surface. These can result in a stronger field enhancements between two particles. Hence, hemispheres would be more suited for biosensing with this scheme than spheres, and in the preparation of NP chain samples it would be desirable to use techniques that produce hemispherical NPs (such as organometallic chemical vapor deposition) than those that produce spherical NPs (such as colloidal casting). 

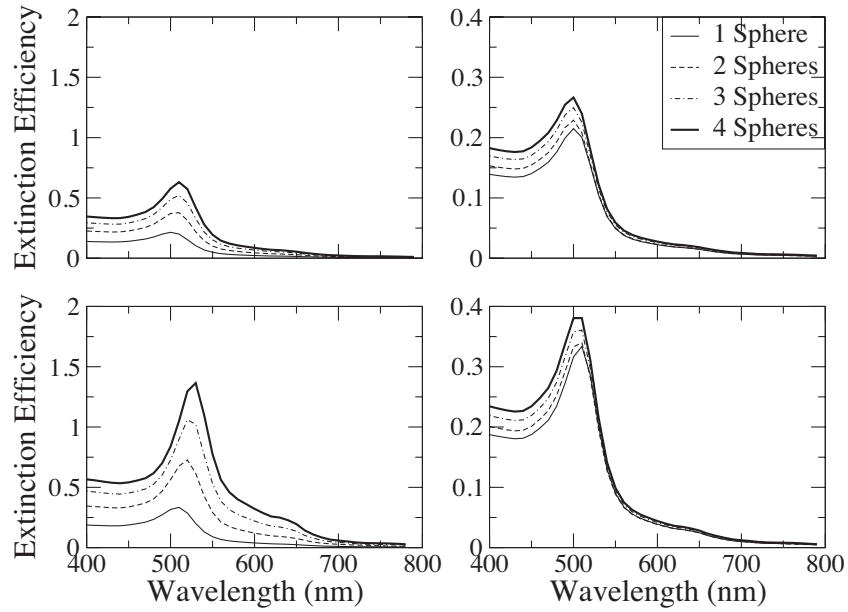

FIG. 4. Extinction efficiency vs wavelength for a GNP chain with different numbers of spherical particles excited by light propagating along the waveguide surface and perpendicular to the chain. Light polarization is either along the interparticle axis ( $s$-polarization) - left two subgraphs, or perpendicular to the waveguide surface ( $p$-polarization) —right two subgraphs. The extinction spectra change from the uncoated spectra (top two panels) to the spectra when coated by a dielectric analyte layer (bottom two panels). The radius of all the spherical NPs is $7 \mathrm{~nm}$ and the thickness of coating layer is $1.75 \mathrm{~nm}$. Interparticle spacing is $17.5 \mathrm{~nm}$ center to center.

\section{B. Refractive index sensitivity}

The sensitivity of this sensing scheme (ratio of peak heights) to the change in refractive index of the dielectric coating was calculated using a four GNP array. As seen in Fig. 5, the shift in the dipole peak with change in refractive index is weak, but the ratio of the two peak heights changes significantly as the refractive index of the coating changes. Thus the sensitivity of this biosensing scheme is expected to be a change in the ratio of two peak heights of 1.35/RIU, where RIU stands for refractive index unit. In an actual implementation, the ratio of two peak heights should be measured for the bare NPs, NPs with recognition sites, and the NPs coated with analyte layer. These three values will confirm the occurence of the sensing reaction, as well as provide a rough measure of the refractive index of the analyte.

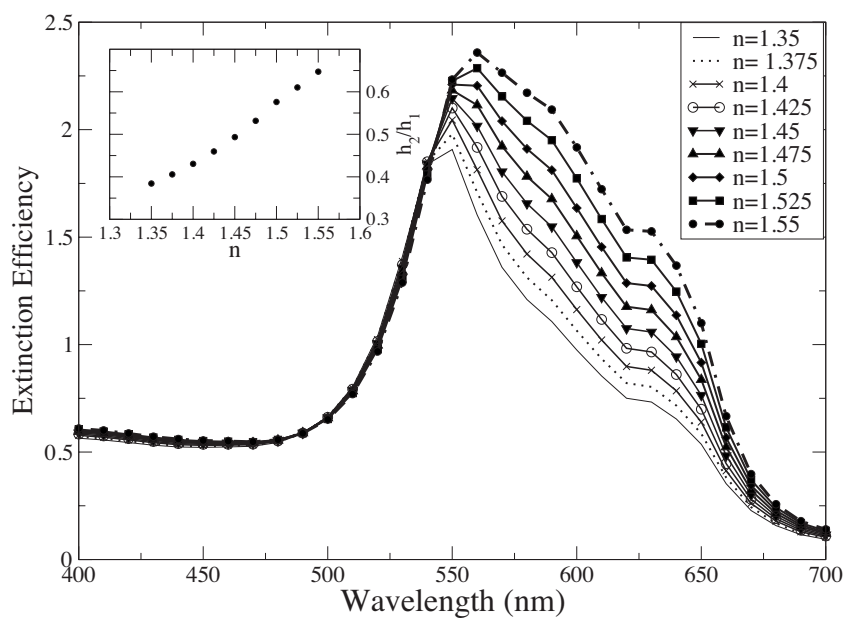

FIG. 5. Sensitivity of the proposed biosensing scheme to change in analyte refractive index. Inset shows that the relative height of the second peak at $\sim 630 \mathrm{~nm}$ to the first peak $\sim(530-550 \mathrm{~nm})$ vs the refractive index of the coating increases roughly linearly with a slope of $1.35 / \mathrm{RIU}$.
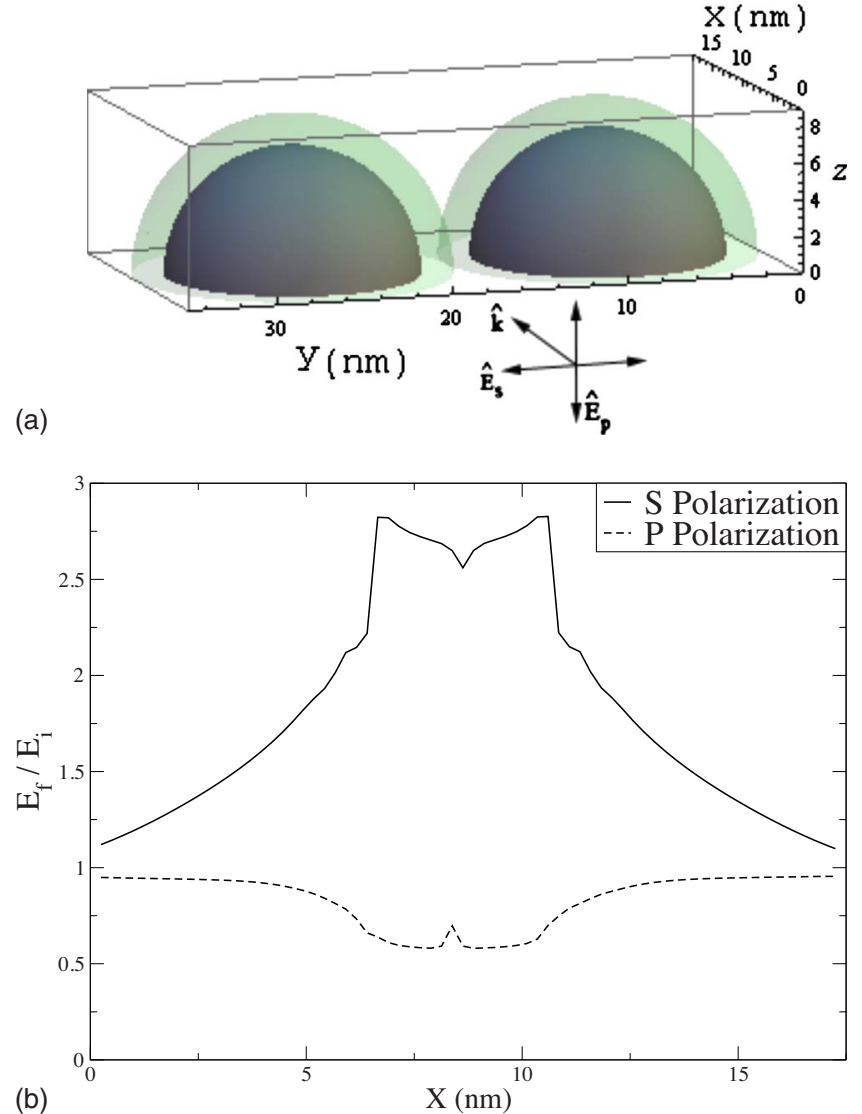

FIG. 6. (Color online) Top: schematic diagram of the target and incident field geometry. Bottom: the change in the magnitude of the electric field due to the dielectric coating $E_{f} / E_{i}$ along the line between the two nanohemispheres at $y=17.5$ and $z=0$. The $s$-polarization case shows field enhancement, whereas the $p$-polarization case shows field supression due to the coating. This effect can be understood by a simple classical model of two interacting dipoles whose lengths increase, but whose strengths decrease due to the dielectric coating.

\section{Physical origin of LSPR spectral broadening}

In an experiment performed with a sample with randomly-spaced NPs (Ref. 16) (not a chain), a weak second peak in the spectrum was experimentally observed. The authors hypothesized that this second "cross talk" peak is a result of the overlap of evanescent fields of two neighboring NPs when the dielectric coating is added. To test this hypothesis, we examine the change in the electric field intensity in the vicinity of the NPs on the waveguide surface for various wavelengths. At a wavelength of $630 \mathrm{~nm}$ (the wavelength at which the second peak appears), the electric field intensity between the two NPs is a maximum. For convenience of display, the results are presented for a dimer, but the key results hold for the chain of NPs. Figure 6 shows that when the dielectric coating is added, and the light is $s$-polarized, the electric field between the two NPs (along the $x$-axis, at $y=17.5$ and $z=0)$ is enhanced by a factor of $2-3$. This is confirms the hypothesis of optical clustering in Ref. 16 as the origin of the second cross talk peak.

It is also seen that when the dielectric coating is added, and the light is $p$-polarized, the electric field is suppressed. This interesting feature can be understood by a simple classical picture: to leading order, the incident light sets up an 


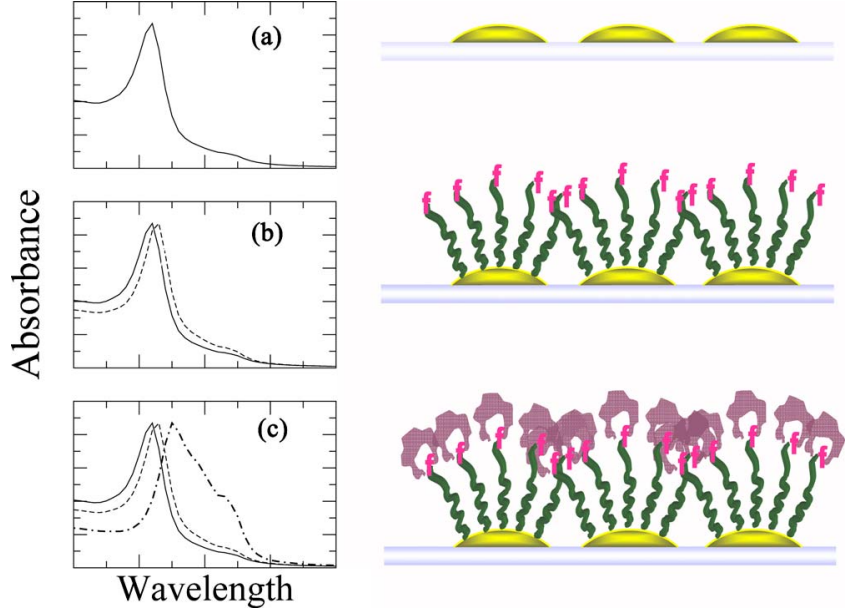

FIG. 7. (Color online) Schematic of proposed NP chain on waveguide sensor. (a) HGNP's immobilized on a transparent substrate give rise to a single particle spectrum. (b) GNPs after functionalization with recognition sites lead to a slight redshift in the spectral peak. (c) Binding the analyte to the recognition sites leads to a huge spectral change-NP-like to nanowirelike-due to the onset of cross-talk.

induced dipole with the axis along the polarization direction of the light. The $s$-polarized light induces dipoles lined endto-end, while the $p$-polarized light induces dipoles that are parallel to each other. When the coating is added, the incident light induces longer (due to the increased size), but weaker (because of the dielectric) dipoles. In the $s$-polarization, the longer dipoles lined end-to-end produce field enhancement between the NPs compared to the uncoated case. But in the $p$-polarization, the parallel arrangement of the weaker induced dipoles leads to a reduction in the field between the NPs.

\section{Proposed NP array biosensor}

In order to propose a biosensor using the above effects, we compare the various competing effects that affect the spectrum, namely, the NP-dielectric coating interaction, the NP-substrate interaction, and the NP-NP interaction. The effect of a coating is to produce a small redshift in the LSPR peak as seen in Fig. 2, and as studied in Ref. 16. Since the NPs are not in contact with the substrate, the effect of the substrate is also to produce a small redshift in the LSPR peak as seen in Fig. 1. The NP-NP interaction produces a large change in the spectrum - the change from a narrow NP-like spectrum to a broad nanowirelike spectrum. We have shown that this effect arises due to the dielectric mediated overlap of the evanescent fields surrounding individual particles, and dominates over the other two effects.

Keeping these sophisticated cross-talk features in mind, we propose a sensor platform based on hemispherical or pancake shaped GNPs immobilized on transparent substrates or waveguides as described schematically in Fig. 7. (a) The NPs are placed to exhibit a pure single NP response and (b) should only show a small LSPR shift by immobilizing a recognition site on them. However, as soon as the analyte binds (c), the cross-talk should set in resulting in a strong change in the localized surface plasmon spectrum. By mea- suring the ratio of two peak heights for cases (a), (b), and (c), one can estimate the refractive index of the analyte.

\section{SUMMARY}

In summary, the results presented in this paper show that the presence of an analyte layer on a chain of hemispherical, GNPs immobilized on a waveguide surface can be detected by observing large change in the evanescent wave absorption spectrum in the $s$-polarization. This effect is a result of the polarization-dependent overlap of the evanescent fields of two adjacent NPs, triggered by the dielectric analyte coating. This scheme provides a measure for determining the sensitivity of a NP sensor-the ratio of the heights of the two peaks depends on the refractive index of the analyte layer. Measuring the ratio of two spectral peaks that are roughly $100 \mathrm{~nm}$ apart provides a higher degree of sensitivity than the traditional peak-shift in order $10 \mathrm{~nm}$ used to determine the sensing reaction. This, and the potential to improve signalto-noise using the two polarization directions underlies the superiority of this proposed platform.

\section{ACKNOWLEDGMENTS}

We gratefully acknowledge research support from NSERC Canada's Discovery Grants Program, the Canada Foundation for Innovation, and the Ontario Research Fund/ Ontario Innovation Trust. T.C. was partly supported by the NSERC Strategic Research Network on Bioplasmonic Systems "BiopSys," and a TEN grant from the Canadian Institute for Photonic Innovations. S.M. was also supported by the Canada Research Chair Program of the Canadian Government. Computations were performed on the Shared Hierarchical Academic Research Computing Network.

${ }^{1}$ C. Yu and J. Irudayaraj, Anal. Chem. 79, 572 (2007).

${ }^{2}$ U. Kreibig and M. Vollmer, Optical Properties of Metal Clusters (Springer, New York, 1995.

${ }^{3}$ C. J. Kiely, J. Fink, M. Brust, D. Bethell, and D. J. Schiffrin, Nature (London) 396, 444 (1998).

${ }^{4}$ A. Ulman, An Introduction to Ultrathin Films (Academic, London, 1991).

${ }^{5}$ A. K. A. Aliganga, A.-S. Duwez, and S. Mittler, Org. Electron. 7, 337 (2006).

${ }^{6}$ S. Link and M. A. El-Sayed, J. Phys. Chem. B 103, 4212 (1999).

${ }^{7}$ T. Jensen, L. Kelly, A. Lazarides, and G. C. Schatz, J. Cluster Sci. 10, 295 (1999).

${ }^{8}$ N. Félidj, J. Aubard, and G. Lévi, Phys. Rev. B 66, 245407 (2002).

${ }^{9}$ K.-H. Su, Q.-H. Wei, X. Zahng, J. J. Mock, D. R. Smith, and S. Schults, Nano Lett. 3, 1087 (2003)

${ }^{10}$ C. Noguez, J. Phys. Chem. C 111, 3806 (2007).

${ }^{11}$ A. J. Haes, D. A. Stuart, S. Nie, and R. P. Van Duyne, J. Flouresc. 14, 355 (2004).

${ }^{12}$ M. J. Adams, An Introduction to Optical Waveguides (Wiley, New York, 1981).

${ }^{13}$ R. G. Hunsperger, Integrated Optics: Theory and Technology, 4th ed. (Springer, New York, 1995).

${ }^{14}$ L. J. Sherry, R. Jin, C. A. Mirkin, G. C. Schatz, and R. P. VanDuyne, Nano Lett. 6, 2060 (2006).

${ }^{15}$ A. K. A. Aliganga, I. Lieberwirth, G. Glasser, A.-S. Duwez, and S. Mittler, Org. Electron. 8, 161 (2007).

${ }^{16}$ P. Rooney, A. Rezaee, S. Xu, T. Manifar, A. Hassanzadeh, G. Podoprygorina, V. Böhmer, C. Rangan, and S. Mittler, Phys. Rev. B 77, 235446 (2008).

${ }^{17}$ G. A. Wurtz, J. S. Im, S. K. Gray, and G. P. Wiederrecht, J. Phys. Chem. B 107, 14191 (2003). 
${ }^{18}$ L. Zhao, K. L. Kelly, and G. C. Schatz, J. Phys. Chem. B 107, 7343 (2003).

${ }^{19}$ V. A. Markel, J. Mod. Opt. 40, 2281 (1993).

${ }^{20}$ E. M. Purcell and C. R. Pennypacker, Astrophys. J. 186, 705 (1973).

${ }^{21}$ B. T. Draine and P. J. Flatau, J. Opt. Soc. Am. A Opt. Image Sci. Vis 11, 1491 (1994).

${ }^{22}$ E. D. Palik, Handbook of Optical Solids (Academic Press, Orlando, 1985), Vol. 1-3.

${ }^{23}$ E. Hao and G. C. Schatz, J. Chem. Phys. 120, 357 (2004).

${ }^{24}$ S. Zou and G. C. Schatz, Chem. Phys. Lett. 403, 62 (2005).
${ }^{25}$ S. Xu, G. Podoprygorina, V. Böhmer, Z. Ding, P. Rooney, C. Rangan, and S. Mittler, Org. Biomol. Chem. 5, 558 (2007).

${ }^{26}$ T. R. Jensen, M. L. Duval, K. L. Kelly, A. A. Lazarides, G. C. Schatz, and R. P. Van Duyne, J. Phys. Chem. B 103, 9846 (1999); M. D. Malinsky, K. L. Kelly, G. C. Schatz, and R. P. Van Duyne, J. Am. Chem. Soc. 123, 1471 (2001); L. J. Sherry, S.-H. Chang, G. C. Schatz, R. P. Van Duyne, B. J. Wiley, and Y. Xia, Nano Lett. 5, 2034 (2005).

${ }^{27}$ M. D. Malinsky, K. L. Kelly, G. C. Schatz, and R. P. Van Duyne, J. Phys. Chem. B 105, 2343 (2001). 